\title{
Nanoscale
}

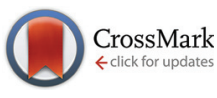

Cite this: Nanoscale, 2014, 6, 14514

\section{Mesoporous titania based yolk-shell nanoparticles as multifunctional theranostic platforms for SERS imaging and chemo-photothermal treatment $\uparrow$}

\begin{abstract}
Weiwei Zhang, ${ }^{\mathrm{a}, \mathrm{b}}$ Yunqing Wang, ${ }^{\star \mathrm{b}}$ Xiuyan Sun, ${ }^{\mathrm{a}}$ Wenhai Wang ${ }^{\mathrm{b}}$ and Lingxin Chen ${ }^{\mathrm{b}}$
Recently surface-enhanced Raman scattering (SERS) imaging guided theranostic nanoplatforms have attracted considerable attention. Herein, we developed novel yolk-shell gold nanorodavoidamesoporous titania nanoparticles (AuNRavoidamTiO 2 NPs) for simultaneous SERS imaging and chemophotothermal therapy. Our work showed three highlighted features: first, we proposed a facile and versatile "up to down" SERS labeling strategy for the drug delivery system, in which "empty carriers" were presynthesized, followed by co-loading of Raman reporters on AuNR and anti-cancer drug doxorubicin (DOX) in $\mathrm{mTiO}_{2}$ in sequence. The acquired SERS signal was strong enough for tracking NPs at both living cells and mice levels. Second, we selected $\mathrm{mTiO}_{2}$ as a novel drug loading material instead of the widely used mesoporous silica $\left(\mathrm{mSiO}_{2}\right)$. The $\mathrm{mTiO}_{2}$ shared satisfactory drug loading and release behavior as $\mathrm{mSiO}_{2}$ but it was chemically inert. This property not only provided a facile way to form a yolk-shell structure but also rendered it with superior structural stability in a biological system. Third, the near infrared (NIR) light absorbing property of the AuNR SERS substrate was also explored for drug release regulation and photothermal treatment. Significantly greater MCF-7 cell killing was observed when treated together with DOX-loaded NPs and NIR laser irradiation, attributable to the synergistic chemo-thermal therapeutic effect. Our results indicated the established SERS labeled yolk-shell NP as a promising theranostic platform and suggested its potential in vivo applications.
\end{abstract}

Received 22nd August 2014 Accepted 29th September 2014 DOI: $10.1039 / \mathrm{c} 4 \mathrm{nr} 04864 \mathrm{~d}$ www.rsc.org/nanoscale chemotherapeutics. Therefore, an enhanced treating efficacy is achieved by the synergistic effects of heat and the drug targetreleased at the tumor sites. ${ }^{5}$

Apart from curing the disease, the function of diagnosis is another major concern in the development of theranostic nanoplatforms. As a newly arising optical detection technique, surface-enhanced Raman scattering (SERS) imaging is considered an ideal diagnostic mode utilized for the construction of theranostic systems. ${ }^{6-10}$ On the one hand, SERS imaging possesses many advantages over traditional NIR fluorescence and magnetic resonance imaging for the high sensitivity, tremendous multiplexing capacity, quantification capability, and high photostability, ${ }^{11,12}$ which have been successfully applied for biological imaging analysis at the level of live-cell, tissues and living small animals. ${ }^{11,13,14}$ On the other hand, many noble metal NP based SERS nanosubstrates such as gold nanorod (AuNR), ${ }^{15,16}$ gold nanocage ${ }^{17}$ and gold nanopopcorn, ${ }^{18}$ are also satisfactory photothermal agents. The bifunction makes it possible to combine SERS image-guided diagnosis, hyperthermia and chemotherapeutics within a relatively simple nanoplatform of few compositions.

Despite noble metal NPs owned the above mentioned bifunctions, they still suffer from two drawbacks as drug delivery systems. The first is their relatively low specific surface area 
limits the loading amount of drugs. The second is that they trend to aggregate after loading drug or entering cells, which induces maximum spectral absorption shifting and the decrease of photothermal efficacy. ${ }^{19}$ To overcome these problems, additional coating was usually applied to increase the colloidal stability and drug molecule storage capacity. For instance, mesoporous silica coated gold nanorod (AuNR@$\mathrm{mSiO}_{2}$ ) was synthesized for anti-cancer drug doxorubicin (DOX) loading and chemo-photothermal treatment for tumors. ${ }^{3,19,20}$ However, many coating conditions, such as the application of organic solvents, high concentration of CTAB or extreme $\mathrm{pH}$ adjustment usually cause Raman reporter to detach from the noble metal NP, resulting in the pre-generated SERS signal to disappear. Therefore, a more versatile nanostructure and a robust SERS signal generation strategy should be explored.

Yolk-shell NPs are a special class of core-shell nanostructures with unique properties of movable cores, interstitial hollow spaces between the movable core and shell sections, which were considered as interesting candidates for developing drug delivery systems. ${ }^{21}$ We speculate that yolk-shell NPs hold great potential for the construction of SERS-based theranostic systems owing to the structural characteristics, in which the noble metal NPs and mesoporous shells could be separated by hollow interiors, thus SERS generation and drug loading could be realized without interference. With this idea in mind, we devised novel multifunctional AuNR@void@m$\mathrm{TiO}_{2}$ yolk-shell NPs, in which NIR light absorbing AuNR inner core is serving as bi-functions of the SERS substrate and the photothermal agent, and the mesoporous titania $\left(\mathrm{mTiO}_{2}\right)$ outer shell being for drug loading. ${ }^{22}$ The synthetic procedure is presented in Scheme 1. In brief, the whole process involved: (1) the synthesis of AuNR@mSiO ${ }_{2}$ NPs; (2) a further coating of $\mathrm{mTiO}_{2}$ on multiple AuNR@mSiO ${ }_{2}$ NPs to form a AuNR@$\mathrm{mSiO}_{2} @ \mathrm{mTiO}_{2}$ nanostructure; (3) the controllable etching of the interior $\mathrm{mSiO}_{2}$ layer with $\mathrm{NaOH}$ to produce AuNR@void@mTiO ${ }_{2}$ yolk-shell NPs; (4) Raman reporter molecules penetrating through the $\mathrm{mTiO}_{2}$ shell, and attaching onto AuNR via a diffusion process under acidic conditions for SERS signal generation; and (5) the loading of DOX in $\mathrm{mTiO}_{2}$ at a neutral $\mathrm{pH}$ environment, endowing NPs with chemotherapeutic functions. Finally, to demonstrate the multifunction, the SERS imaging and chemo-photothermal therapeutic effects were tested upon irradiation of the NIR laser.

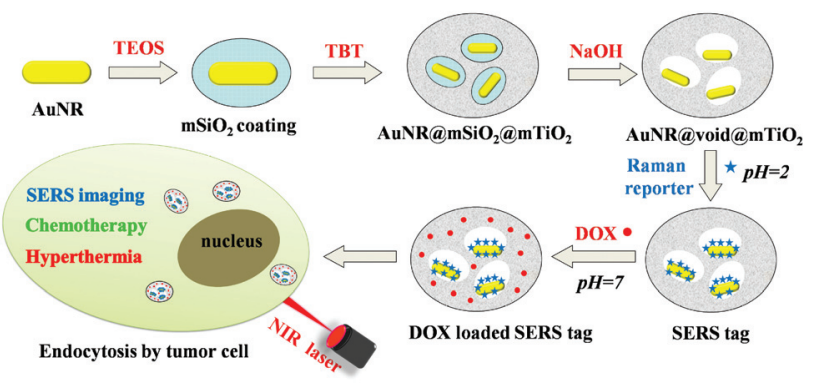

Scheme 1 Synthesis of AuNRavoidamTiO 2 yolk-shell NPs as multifunctional theranostic platforms for cancer treatment.

\section{Experimental}

\subsection{Materials}

Chloroauric acid $\left(\mathrm{HAuCl}_{4} \cdot 3 \mathrm{H}_{2} \mathrm{O}\right)$, silver nitrate $\left(\mathrm{AgNO}_{3}\right)$, sodium borohydride $\left(\mathrm{NaBH}_{4}\right)$, sodium hydroxide $(\mathrm{NaOH})$, L-ascorbic acid (AA), crystal violet, tetraethoxyorthosilicate (TEOS) and tetrabutyl titanate (TBT) were purchased from Sinopharm Chemical Reagent Co., Ltd. Nonionic surfactant Lutensol ON50 was obtained from BASF. Cetyltrimethyl ammonium bromide (CTAB), doxorubicin hydrochloride (DOX), 3,3'-diethylthiadicarbocyanine iodine (DTDC) and 3,3'diethylthiatricarbocyanine iodide (DTTC) were obtained from Sigma-Aldrich. 5-Carboxyfluorescein diacetate (CFDA) and propidium iodide (PI) were obtained from Aladdin Industrial Co., Ltd. MTT (3-(4,5-dimethylthiazol-2-yl)-2,5-diphenyltetrazolium bromide) was purchased from Invitrogen. RPMI-1640 medium, fetal bovine serum, penicillin-streptomycin solution and trypsin-EDTA solution were purchased from Thermo Scientific. Deionized water was used in all the experiments.

\subsection{Characterization}

The TEM images were acquired on a JEM-1400 transmission electron microscope (JEOL, Japan). The SEM images were obtained on an S-4800 field emission scanning electron microscope (Hitachi, Japan). UV/Vis/NIR absorption spectra were recorded on a Thermo Scientific NanoDrop 2000/2000C spectrophotometer. SERS spectra were recorded using a DXR Raman Microscope (Thermo Fisher, USA). A $632.8 \mathrm{~nm}$ He:Ne laser was focused by a $\times 10$ and $\times 50$ microscope objective for sample solution and cell measurement, respectively, with a power of $2 \mathrm{~mW}$. Confocal laser scanning microscopy (CLSM) was performed on an Olympus Fluoview FV1000 (Japan). The hydrodynamic diameters and zeta potentials of the NPs were measured on a Zetasizer Nano ZS90 (Malvern, U.K.) in water medium.

\subsection{Preparation of AuNRs}

AuNRs were synthesized using the seed-mediated growth method. ${ }^{23}$ Briefly, the seed solution was prepared by reducing $\mathrm{HAuCl}_{4}(0.5 \mathrm{mM}, 2 \mathrm{~mL})$ in CTAB $(0.2 \mathrm{M}, 2 \mathrm{~mL})$ with freshly prepared ice-cold $\mathrm{NaBH}_{4}(10 \mathrm{mM}, 0.24 \mathrm{~mL})$. After $2 \mathrm{~h}, 3.6 \mathrm{~mL}$ of the resulting seed solution was added into a growth solution of $\mathrm{HAuCl}_{4}(23 \mathrm{mM}, 13 \mathrm{~mL}), \mathrm{CTAB}(0.2 \mathrm{M}, 200 \mathrm{~mL}), \mathrm{AgNO}_{3}$ (4 mM, $11.2 \mathrm{~mL})$, and AA (0.08 M, $5 \mathrm{~mL})$. The mixture was left overnight at $27-30^{\circ} \mathrm{C}$.

\subsection{Preparation of AuNR@mSiO ${ }_{2} \mathrm{NPs}$}

AuNR@mSiO ${ }_{2}$ NPs were synthesized according to a published procedure with some minor modifications. ${ }^{24}$ The as-prepared AuNRs $(40 \mathrm{~mL})$ were centrifuged $(9500 \mathrm{r}, 25 \mathrm{~min})$ and dispersed in water $(20 \mathrm{~mL})$, followed by addition of $\mathrm{NaOH}(0.1 \mathrm{M}$, $200 \mu \mathrm{L})$ under stirring. Then, $20 \%$ TEOS in ethanol $(180 \mu \mathrm{L})$ was added three times at $30 \mathrm{~min}$ intervals under mixing. The resulting solution was further allowed to react for $48 \mathrm{~h}$ at 26-28 ${ }^{\circ} \mathrm{C}$. The obtained AuNR@mSiO ${ }_{2}$ core-shell NPs were then collected and washed twice with water (9000 r, $15 \mathrm{~min})$, 
once with ethanol (8500 r, $15 \mathrm{~min})$, and then dispersed in $20 \mathrm{~mL}$ ethanol.

\subsection{Preparation of AuNR@mSiO ${ }_{2} @ \mathrm{mTiO}_{2} \mathrm{NPs}$}

A typical procedure for coating AuNR@mSiO ${ }_{2}$ particles with $\mathrm{mTiO}_{2}$ is as follows. ${ }^{25,26}$ Firstly, a $0.1 \mathrm{M}$ aqueous solution of Lutensol ON50 $(4 \mu \mathrm{L})$ was dissolved in a pre-mixed dispersion of AuNR@mSiO $\mathrm{m}_{2}$ core particles $(200 \mu \mathrm{L})$ in ethanol $(300 \mu \mathrm{L})$. Then, $500 \mu \mathrm{L}$ of ethanol was mixed with TBT $(10 \mu \mathrm{L})$. This diluted titania precursor solution was then added to the dispersion of core particles, followed by vigorous shaking. The mixture was sonicated for at least $20 \mathrm{~min}$ in an ultrasound bath before being left overnight to allow the reaction to go to completion. The AuNR@mSiO $@_{2} @ \mathrm{mTiO}_{2} \mathrm{NPs}$ were washed twice (5000 r, $5 \mathrm{~min}$ ) with ethanol and water and then dispersed in $250 \mu \mathrm{L}$ water.

\subsection{Preparation of AuNR@void@mTiO $\mathrm{TPs}_{2}$}

A NaOH solution $(0.5 \mathrm{M}, 250 \mu \mathrm{L})$ was added to the AuNR@$\mathrm{mSiO}_{2} @ \mathrm{mTiO}_{2} \mathrm{NP}$ solution, and the mixture was kept for $2 \mathrm{~h}$ to selectively remove the $\mathrm{mSiO}_{2}$. The resulting AuNR@void@m $\mathrm{miO}_{2}$ yolk-shell particles were isolated by centrifugation and washed three times with water (4000 r, $5 \mathrm{~min}$ ), and finally dispersed in $500 \mu \mathrm{L}$ water.

\subsection{SERS signal generation}

The pH of AuNR@void@mTiO ${ }_{2}$ NP solution $(500 \mu \mathrm{L})$ was adjusted to about 2 with concentrated hydrochloric acid before the addition of the Raman reporter molecule DTDC $\left(10^{-4} \mathrm{M}\right.$, $50 \mu \mathrm{L})$. The mixture was kept for $30 \mathrm{~min}$ and then centrifuged (4000 r, $5 \mathrm{~min}$ ) and washed twice with water and then dispersed in $500 \mu \mathrm{L}$ water for SERS signal measurement.

\subsection{Anti-cancer drug (DOX) loading}

DTDC labeled NPs $(500 \mu \mathrm{L})$ were centrifuged and redispersed into a solution of DOX in water $\left(2 \times 10^{-4} \mathrm{M}, 500 \mu \mathrm{L}\right)$. After different incubation times (5, 10, 20, 30, 40, 60 and $90 \mathrm{~min}$ ), the NPs were centrifuged and the supernatant was subjected to UV/Vis analysis to determine the amount of loaded DOX. A stock solution of DOX $\left(10^{-4} \mathrm{M}\right)$ was used as a standard and was serially diluted to concentrations of 5.0-100 $\times 10^{-6} \mathrm{M}$ in water. DOX solutions of different concentrations were measured at $480 \mathrm{~nm}$ and a linear fit of the data was created and used as a standard curve for the absorption against DOX concentrations.

\subsection{Photothermal properties}

$1 \mathrm{~mL}$ of the AuNR@void@mTiO 2 NP solution $\left(0.2 \mathrm{mg} \mathrm{mL}{ }^{-1}\right)$ filled in a $1.5 \mathrm{~mL}$ Eppendorf tube was irradiated for $10 \mathrm{~min}$ by a NIR laser (785 nm, $400 \mathrm{~mW})$ at a distance of $1 \mathrm{~cm}$ from the side of the solution. A thermoindicator was used to monitor the temperature change. Water was utilized as a negative control and AuNR with the same concentration was utilized as a positive control.

\subsection{In vitro drug release}

$1.0 \mathrm{~mL}$ of DOX-loaded NP solution in PBS buffers with different pHs $(5.0,7.4)$ was agitated at room temperature. The mixture was centrifuged at different time intervals. The supernatant was collected and the same volume of fresh buffer was added back to the residual. The amount of the released drug in the supernatant was measured using a UV-Vis spectrometer at $480 \mathrm{~nm}$. A similar procedure was applied in the NIR laser controlled drug release study. The NP buffer solution ( $\mathrm{pH}$ 5.0) was irradiated with a NIR laser $(785 \mathrm{~nm}, 400 \mathrm{~mW})$ for $6 \mathrm{~min}$. The supernatant was collected for UV-Vis measurement to study the DOX release behavior.

\subsection{Mesoporous shell stability}

AuNR@mSiO ${ }_{2}$ and AuNR@void@mTiO ${ }_{2}$ solution sample ( $1 \mathrm{~mL}$ for each) with the same AuNR concentration was centrifuged and further dispersed into pH 7.4 PBS buffer $(0.01 \mathrm{M}$, $1 \mathrm{~mL}$ ). The mixtures were gently stirred at a constant temperature of $37{ }^{\circ} \mathrm{C}$. The NPs were centrifuged and collected at different intervals for morphology observation via a SEM.

\subsection{Cell culture}

A breast-cancer-cell line (MCF-7) was grown as a monolayer in a humidified incubator at $37{ }^{\circ} \mathrm{C}$ in air/ $\mathrm{CO}_{2}$ (95:5) in an RPMI-1640 medium that was supplemented with $10 \%$ fetal bovine serum. For all experiments, the cells were harvested by using trypsin and were resuspended in fresh medium before plating.

\subsection{Cell imaging}

MCF-7 cells $\left(2.0 \times 10^{4}\right)$ were seeded onto glass cover-slips in a 24-well plate with $540 \mu \mathrm{L}$ culture medium to allow the cells to attach. Then a $60 \mu \mathrm{L}$ DOX-loaded yolk-shell NP solution $(20 \mu \mathrm{g}$ $\mathrm{mL}^{-1}$, with $1 \mu \mathrm{M}$ DOX) was added into the cells. After incubation for different times, the cell monolayer on the cover-slip was repeatedly washed with PBS to remove the remaining particles and the dead cells and then sealed with a glass microscope slide. Observations were performed by confocal laser scanning microscopy and Raman microscopy.

\subsection{In vivo SERS detection}

$100 \mu \mathrm{L}$ of the DOX-loaded NP solution was injected subcutaneously in the abdomen of an anaesthetized Kunming mouse. The subcutaneous SERS spectrum was detected using an Agility Raman spectrometer (BaySpec, USA). The laser power was $200 \mathrm{~mW}$ and the integration time was $1 \mathrm{~s}$. The control spectrum was taken in an area away from the injected site.

\subsection{Therapeutic effect assessment}

$8 \times 10^{3}$ MCF-7 cells were plated in 96-well plates and incubated for $24 \mathrm{~h}$ to allow the cells to attach. AuNR@void@mTiO yolk- $^{-}$ shell NPs were added into the cells to form NP suspensions in culture media with different concentrations $(0,1,5,10,20,40$,

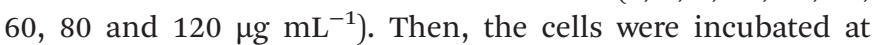


$37^{\circ} \mathrm{C}$ for another $24 \mathrm{~h}$. Cell viability of the blank nanocarriers (before loading drugs) was evaluated using an MTT assay. Thereafter, in a separate experiment, the cells were exposed to free DOX and DOX-loaded NPs with a NP concentration of 10 $\mu \mathrm{g} \mathrm{mL} \mathrm{m}^{-1}$ and a DOX concentration of $1 \mu \mathrm{M}$. The cells were or were not irradiated by the NIR laser $\left(6.25 \mathrm{~W} \mathrm{~cm}^{-2}\right)$ with illumination for $5 \mathrm{~min}$ after $6 \mathrm{~h}$ of drug incubation. Then the cells were incubated at $37{ }^{\circ} \mathrm{C}$ for another $24 \mathrm{~h}$. The cells were washed with PBS buffer, submerged in $500 \mu \mathrm{L}$ PBS, examined under a fluorescence microscope using a live-cell staining procedure consisting 5-carboxyfluorescein diacetate (CFDA, 100 $\mu \mathrm{g} \mathrm{mL} \mathrm{m}^{-1}$ in PBS, $10 \mu \mathrm{L}$ ) and propidium iodide (PI, $30 \mu \mathrm{g} \mathrm{mL}$ in PBS, $7.5 \mu \mathrm{L}$ ). Non-fluorescent CFDA can be converted to green fluorescent 5-carboxyfluorescein (with $495 \mathrm{~nm}$ laser irradiation) by esterases in living cells. Membrane impermeant PI enters dead cells and displayed enhanced red fluorescence when it binds to DNA/RNA. Then, the effects of exposure to laser, DOX and the multifunctional NPs were quantitatively evaluated by MTT assays.

\section{Results and discussion}

\subsection{Synthesis of AuNR@void@mTiO 2 yolk-shell NPs}

The TEM image of AuNR (Fig. 1a) showed that the average length and width were around $43 \mathrm{~nm}$ and $12 \mathrm{~nm}$, respectively (about 3.6:1 aspect ratio). The TEM images of AuNR@mSiO NPs (Fig. 1b) indicated that each AuNR was coated with a uniform $\mathrm{mSiO}_{2}$ shell of $15 \mathrm{~nm}$ thickness and these core-shell NPs were uniform and well-dispersed. During the $\mathrm{mTiO}_{2}$ coating process, it was found that the concentration of AuNR@mSiO ${ }_{2}$ core NPs affected the shape of the products. When the amount of core NPs was $200 \mu \mathrm{L}$ in a $1.0 \mathrm{~mL}$ reaction mixture, the resulting $\mathrm{mTiO}_{2}$ coated NPs were sphere like with the particle size around $250 \mathrm{~nm}$ (Fig. 1c and d). Dynamic light scattering measurement revealed a reasonable, but a little larger hydrodynamic diameter in the range of $250 \mathrm{~nm}$ to $450 \mathrm{~nm}$ (Fig. S1 $\dagger$ ). When the volume of core NPs was increased to $500 \mu \mathrm{L}$, a nonuniform "worm-shaped" structure was obtained (Fig. S2a and $\mathrm{b} \dagger$ ). It had been reported that a $\mathrm{mTiO}_{2}$ structure synthesized with Lutensol ON50 had a BarrettJoyner-Halenda adsorption average pore diameter of $1.68 \mathrm{~nm},{ }^{27}$ which provided a precondition for the subsequent $\mathrm{NaOH}$ penetration and the resulting $\mathrm{mSiO}_{2}$ etching process. Under basic conditions, $\mathrm{mSiO}_{2}$ in the middle layer was dissolved by weakening the siloxane bonds and coordinating $\mathrm{OH}^{-}$ ions to $\mathrm{Si}$ atoms, while the outer $\mathrm{mTiO}_{2}$ shell was kept compact due to its chemical inertness, ${ }^{28,29}$ thus a yolk-shell NP structure could be formed. A high-magnification TEM image of the nanosphere NP in Fig. 1d showed pale rings around multiple AuNRs, indicating gaps between AuNR and $\mathrm{mTiO}_{2}$. For nanoworm NPs the yolk-shell structure was much easier to be observed due to the relatively thin $\mathrm{mTiO}_{2}$ shell (Fig. S2c $\dagger$ ). This yolk-shell NP structure was simply achieved by tactfully taking advantage of the stability difference of $\mathrm{mSiO}_{2}$ and $\mathrm{mTiO}_{2}$, avoiding multi-steps or the application of
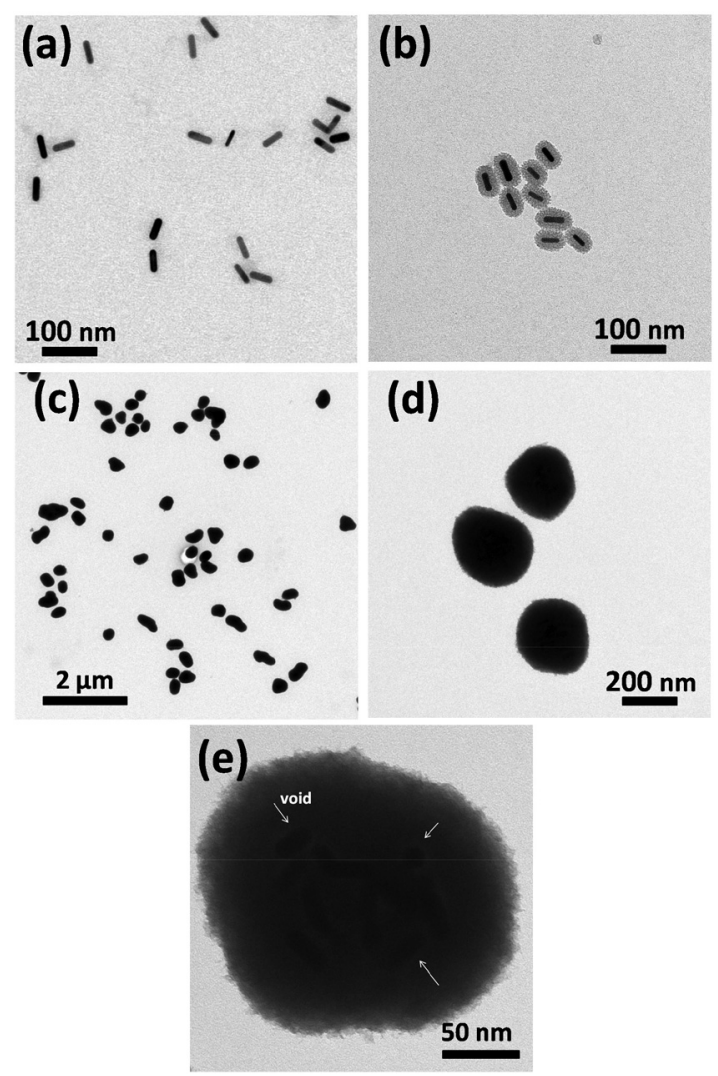

Fig. 1 TEM images of AuNR (a), AuNRamSiO 2 (b) and sphere like AuNRamSiO ${ }_{2} \mathrm{amTiO}_{2} \mathrm{NPs}(\mathrm{c}, \mathrm{d})$. (e) shows a high resolution TEM image of a AuNR@void@mTiO 2 yolk-shell nanosphere. The white arrows indicated the gaps between AuNRs and $\mathrm{mTiO}_{2}$.

high temperature and non-green etching agents. ${ }^{30}$ Considering the demand of uniformity and drug loading capacity of nanomedicine, sphere like yolk-shell structures with a thicker $\mathrm{mTiO}_{2}$ shell was used in the following study.

\subsection{Optical characterization of AuNR@void@m $\mathrm{TiO}_{2} \mathrm{NPs}$}

As shown in Fig. 2a, the longitudinally localized surface plasmon resonance (SPR) band of AuNR@ $\mathrm{mSiO}_{2}$ red shifted about $20 \mathrm{~nm}$ (from $764 \mathrm{~nm}$ to $784 \mathrm{~nm}$ ) compared with the absorption spectrum of bare AuNR. This could be explained by the fact that the local refractive index of the silica shell (1.45) was larger than water (1.33). ${ }^{31}$ Interestingly, after dissolving $\mathrm{mSiO}_{2}$, the "spaced" $\mathrm{mTiO}_{2}$ shell had a similar red-shift effect as the adhered $\mathrm{mSiO}_{2}$ to AuNR and yolk-shell NPs still showed a longitudinal SPR absorption at $784 \mathrm{~nm}$. Meanwhile, a strong end-absorption at $272 \mathrm{~nm}$ appeared, which was attributed to UV light absorption of $\mathrm{mTiO}_{2}$ coating.

To render the NP SERS signals, we further investigated the Raman reporter labeling strategy. In general, the signal of SERS tags is usually produced following the sequence of noble metal NP preparation, Raman reporter attachment, and surface protection, which was regarded as a "bottom-up" way. However, for the production of SERS labeled multifunctional nanomedicine, this sequence would induce problems because 

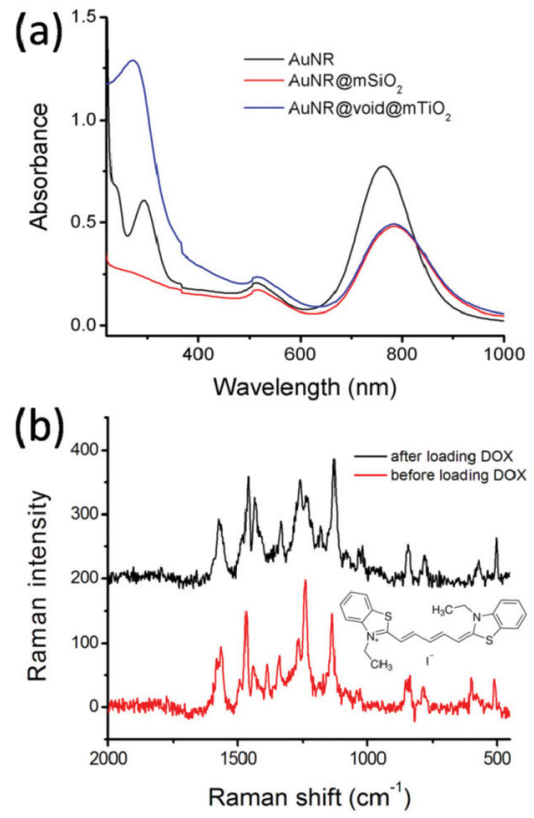

Fig. 2 (a) UV/Vis/NIR absorption spectra of AuNR, AuNR@mSiO 2 and AuNRavoidamTiO ${ }_{2}$ NPs in water. (b) SERS spectra of DTDC labeled AuNRavoidamTiO ${ }_{2}$ before and after loading DOX. The inset shows the molecular structure of Raman reporter DTDC.

the pre-generated SERS disappeared because several severe synthesis conditions (high concentration of CTAB at the $\mathrm{mSiO}_{2}$ coating process and $\mathrm{NaOH}$ etching stage) could detach Raman reporters from the metal substrates. Herein, we proposed a versatile "up-down" strategy, in which SERS nanosubstrates were pre-stabilized by polymers ${ }^{32}$ or mesoporous materials, ${ }^{33,34}$ followed by Raman reporters' subsequent mixing and infiltration step. In our case, it was crucial to load the Raman reporter DTDC and the therapeutic drug DOX at appropriate positions of the nanoplatform (on AuNR and in $\mathrm{mTiO}_{2}$, respectively), which determined the optical and therapeutic properties. To achieve this goal, we firstly tried a simple signal generation condition to mix the yolk-shell NPs and the DTDC solution in neutral pH. As shown in Fig. S3a, $\uparrow$ the apparent color change of the NP solution from brown to purple indicated that a large amount of DTDC was adsorbed in $\mathrm{mTiO}_{2}$ via a strong electrostatic interaction. The strong fluorescence background from DTDC overwhelmed the SERS signals (Fig. S3b†). In order to decrease this unwanted electrostatic interaction, we tested another way to adjust the $\mathrm{pH}$ of the NP solution to about 2 before incubation with DTDC. As shown in Fig. 2b, after washing and dispersing NPs in water, a characteristic and "clean” DTDC SERS signal was obtained from the NP solution without fluorescence background under irradiation of $632.8 \mathrm{~nm}$ laser. ${ }^{35}$ Two reasons contributed to this fortunate result: (1) under the acidic conditions, most Ti-OH groups would be protonated, which could prevent the adsorption and facilitate the positively charged DTDC to penetrate through the $\mathrm{mTiO}_{2}$ shell to access the AuNR surface. This fluorescence quenched state suggested the loaded DTDC was mostly on the surface of AuNR but not in $\mathrm{mTiO}_{2}{ }^{7}$ (2) AuNR still maintained strong affinity to the Raman reporter via stable $\mathrm{Au}-\mathrm{N}$ and $\mathrm{Au}-\mathrm{S}$ bonds in such acidic medium. Therefore, after DTDC attachment, the SERS labeled yolk-shell NPs could still preserve the unoccupied $\mathrm{mTiO}_{2}$ shells for further drug molecule loading. We also tested other two commonly used Raman reporters, i.e., crystal violet and DTTC, for SERS signal generation of our yolk-shell NPs. As shown in Fig. S4, $\dagger$ the final products exhibited characteristic Raman signature of the corresponding Raman reporter, indicating that the method was versatile provided the chemical structure of the Raman reporter was stable under acidic conditions. To test whether the drug loading affects the SERS signal, we loaded DOX in $\mathrm{mTiO}_{2}$ shell at neutral $\mathrm{pH}$, and it did not cause any apparent variation of the SERS profile (Fig. 2b, the red line).

\subsection{Photothermal properties of NPs}

The AuNR@void@mTiO ${ }_{2}$ NPs exhibited a high NIR absorption from 700 to $900 \mathrm{~nm}$, making it a potential photothermal treatment agent. As shown in Fig. 3a, under irradiation of a $785 \mathrm{~nm}$ NIR laser at a power of $400 \mathrm{~mW}$ for $10 \mathrm{~min}$, pure AuNR showed the best heat converting efficiency, with the temperature increase from $25{ }^{\circ} \mathrm{C}$ to $55{ }^{\circ} \mathrm{C}$. Our AuNR@void@m $\mathrm{mTiO}_{2} \mathrm{NP}$ solution also showed dramatic temperature changes from $25^{\circ} \mathrm{C}$ to $51{ }^{\circ} \mathrm{C}$. AuNR@void@mTiO ${ }_{2} \mathrm{NP}$ showed a little weaker efficacy than AuNR, which was because the $\mathrm{mTiO}_{2}$ shell around the AuNR affected light absorption and local heat diffusion in solution to some extent. As a negative control, the temperature of water was stable at $25{ }^{\circ} \mathrm{C}$. After 10 min treatment, the absorption spectrum of the samples was
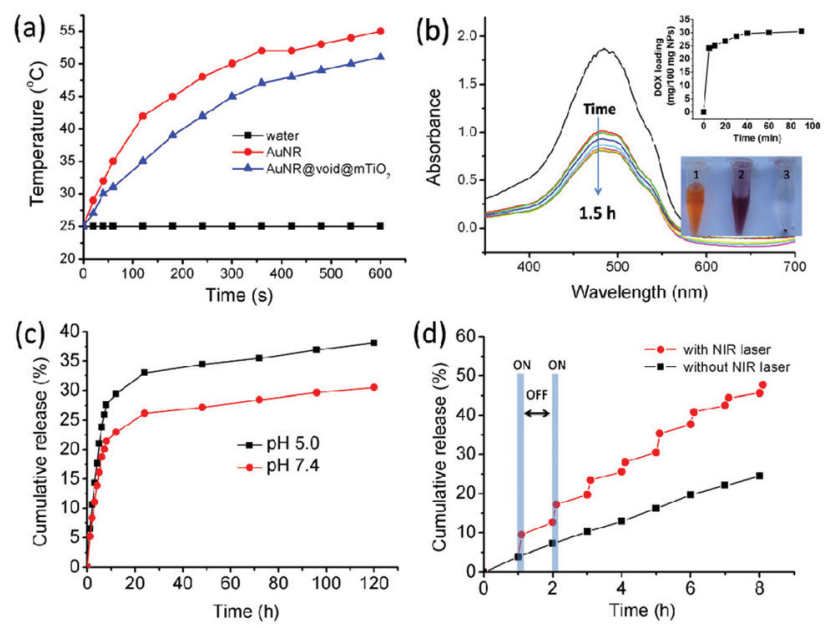

Fig. 3 (a) Heating curves of water, AuNR and AuNRavoidamTiO 2 NP solutions with the same concentration of Au NR. The power of $785 \mathrm{~nm}$ laser was $400 \mathrm{~mW}$ and the distance to the Eppendorf tube filled with NP solution was $1 \mathrm{~cm}$. (b) UV/Vis absorption spectra of DOX in separated supernatant solutions after various loading times with AuNRavoida$\mathrm{mTiO}_{2} \mathrm{NPs}$. Inset: loading profile of DOX with time and photographs of DOX solution (left), DOX-loaded NPs before (middle) and after (right) centrifugation. (c) DOX release profiles from DOX-loaded NPs at different PBS solutions. (d) Comparison of DOX release profiles from DOX-loaded NPs with and without $785 \mathrm{~nm}$ NIR laser at PBS buffer ( $\mathrm{pH} 5.0$ ). 
unchanged, indicating that the AuNR cores were stable and did not melt under laser irradiation conditions.

\subsection{Drug loading and release behaviors}

Recently, $\mathrm{mTiO}_{2}$ NPs had been found to be promising vehicles for intracellular drug delivery, ${ }^{22,26,36}$ and we further investigated the drug loading and release behaviors of the $\mathrm{mTiO}_{2}$ shell in the yolk-shell NPs. Fig. 3b shows the UV/Vis absorption spectra of an aqueous solution of DOX before and after the interaction with SERS labeled NPs. The absorption intensity of DOX decreased significantly, indicating that the DOX had been stored in NPs. The loaded efficiency was estimated to be $30.5 \mathrm{mg}$ DOX per $100 \mathrm{mg}$ NPs. The high loading capacity was attributed to strong electrostatic interactions between the positively charged DOX and the negatively charged $\mathrm{mTiO}_{2}$ of large surface area under neutral conditions. The zeta potential of blank NPs determined in water was $-44.5 \mathrm{mV}$, after adsorbing DTDC it was nearly stable at $-41.3 \mathrm{mV}$, whereas further loading of DOX made it sharply increase to $-28.3 \mathrm{mV}$ (Fig. S5 $†$ ). This indicated that during the SERS generation process DTDC did not occupy the mesopores of $\mathrm{mTiO}_{2}$, while DOX did in the drug loading process.

The in vitro drug release behavior was studied in $\mathrm{pH} 7.4$ and 5.0 PBS buffers to simulate a neutral environment of blood circulation and acidic conditions in cellular endosomes, respectively. As shown in Fig. 3c, DOX release was a little faster at $\mathrm{pH} 5.0$ than that at $\mathrm{pH}$ 7.4, which might be because the solubility of DOX decreased with the increase of $\mathrm{pH}$ in aqueous solution and the poor solubility lagged the drug release process. ${ }^{37}$ Furthermore, we used a $785 \mathrm{~nm}$ laser $(400 \mathrm{~mW})$ to irradiate the DOX-loaded NP solution ( $\mathrm{pH}$ 5.0) for $6 \mathrm{~min}$ at the beginning of every $1 \mathrm{~h}$. As shown in Fig. 3d, the drug release became faster, which increased from $24.5 \%$ (without laser treatment) to $47.7 \%$ in $8 \mathrm{~h}$, and illustrated an apparent laser "switch-on" release phenomenon. AuNR encapsulated in NPs converted NIR light to local heating, which dissociated the strong interactions between $\mathrm{DOX}$ and $\mathrm{mTiO}_{2}$ thus more DOX molecules were released.

\subsection{Structural stability}

Mesoporous NPs are good at drug loading due to the loose structure full of nanosized mesopores. In contrast, such a structure suffers from the drawback of chemical and mechanical stabilities compared with the "solid" counterpart. It has been reported that $\mathrm{mSiO}_{2}$ can be severely degraded under simulated physiological ( $\mathrm{pH} 7.4$ PBS buffer) conditions, ${ }^{38}$ which not only influence its drug delivery capacity but also leave a potential risk for the biocompatibility. Lately researchers have found that $\mathrm{mSiO}_{2}$ NPs showed more apparent in vivo toxicity than solid $\mathrm{SiO}_{2} \mathrm{NPs},{ }^{39}$ and degradation of $\mathrm{mSiO}_{2}$ and the resulting products might be clues which explain this phenomenon. Considering the chemically inert feature of $\mathrm{mTiO}_{2}$, we speculated that $\mathrm{mTiO}_{2}$ had better stability under physiological conditions. To prove this, our AuNR@void@$\mathrm{mTiO}_{2}$ NPs were dispersed in pH 7.4 PBS buffer and gently stirred for $12 \mathrm{~h}$ at $37^{\circ} \mathrm{C}$. For comparison AuNR@mSiO ${ }_{2}$ NPs
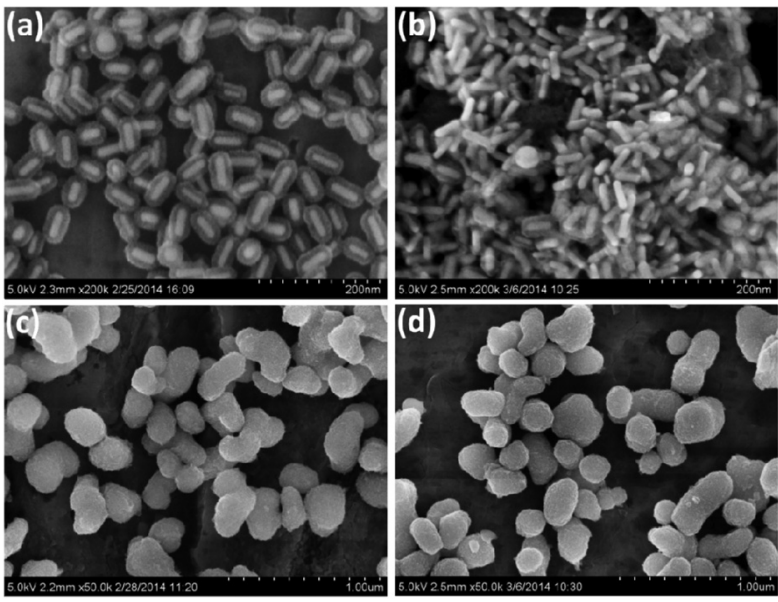

Fig. $4 \mathrm{SEM}$ images of AuNR@mSiO 2 NPs $(a, b)$ and $\mathrm{AuNR}$ avoid@amTiO 2 NPs $(c, d)$ before $(a, c)$ and after $(b, d)$ stirring in $\mathrm{pH} 7.4$ PBS buffer (0.01 M) at $37^{\circ} \mathrm{C}$ for $12 \mathrm{~h}$.

were also subjected to the same treatment. As expected, SEM images showed that $\mathrm{mSiO}_{2}$ coated AuNR@mSiO 2 NPs dissolved to a large extent after the treatment, resulting in aggregated bare AuNR (Fig. 4a and b), a condition consistent with that of the reported work, ${ }^{38}$ whereas the structure of AuNR@void@ $\mathrm{mTiO}_{2} \mathrm{NP}$ still kept compact (Fig. 4c and d). The difference owing to $\mathrm{Ti}-\mathrm{OH}$ was much stable than $\mathrm{Si}-\mathrm{OH}$ in a weak basic atmosphere. Taking serial experimental results into account, it was essential to reexamine the stability, biocompatibility, and the drug release mechanism of the $\mathrm{mSiO}_{2}$ as a wellaccepted drug delivery material. In contrast, our $\mathrm{mTiO}_{2}$ based nanomedicine held great advantage from the aspect of mechanical stability, which was first reported to the best of our knowledge.

\subsection{Optical imaging}

To verify the NPs' intracellular drug delivery ability, confocal laser scanning microscopy (CLSM) images of MCF-7 cells were taken after treatment with DOX-loaded NPs at different incubation times. As shown in Fig. 5a, very weak red fluorescence that resulted from DOX could be visualized in the cytoplasm after co-incubation for $1 \mathrm{~h}$. When the incubation time was increased to $3 \mathrm{~h}$, a much enhanced fluorescence was observed, indicating that more NPs were taken up by tumor cells via endocytosis. Furthermore, we investigated the intracellular SERS performance of the drug-loaded NPs by a Raman microscope equipped with a $632.8 \mathrm{~nm}$ laser. Fig. 5b shows the bright field images of the MCF-7 cells after NP incubation. It was obvious that the NPs mainly distributed in cytoplasmic regions and almost no particles were observed from the nucleus, because the NPs were not small enough to penetrate into the nucleus. SERS spectra from different locations of one cell showed the strong signals that appeared at the cytoplasm while the signal at the nucleus was nearly undetected. Besides, the characteristic Raman signal of DTDC remained robust and 

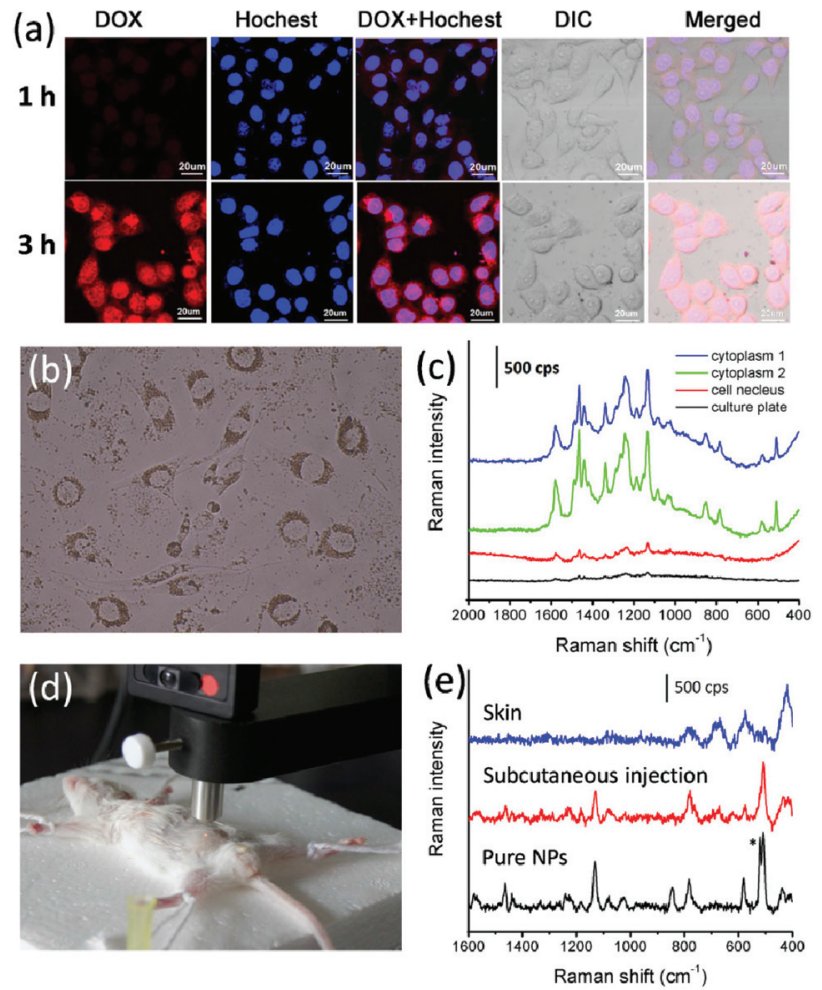

Fig. 5 (a) Confocal laser scanning microscopy images of MCF-7 cells taken after treatment with DOX-loaded NPs at different incubation times. Hoechst 33342 was used for cell nuclei staining (blue fluorescence). Red fluorescence in the MCF-7 cells originated from DOX. (b) Bright field images of MCF-7 cells after incubating DOX-loaded AuNRavoidamTiO $\mathrm{NPs}_{2}$ for $4 \mathrm{~h}$ and (c) corresponding Raman spectra at four different locations across one NP filled MCF-7 cell under $632.8 \mathrm{~nm}$ irradiation. (d) Photograph of a Kunming rat subcutaneously injected with $100 \mu \mathrm{L}$ of DOX-loaded NP solution $\left(0.2 \mathrm{mg} \mathrm{mL}^{-1}\right)$ under $785 \mathrm{~nm}$ excitation (200 mW). (e) SERS spectra from pure NP, the NP injected site and the blank skin. The peak at $520 \mathrm{~cm}^{-1}$ indicated by an asterisk originated from the silicon wafer.

no obvious interference Raman peaks were obtained due to the protection of the $\mathrm{mTiO}_{2}$ layer (Fig. 5c).

The in vivo SERS imaging performance of the NPs was evaluated in a mouse (Fig. 5d). $200 \mu \mathrm{L}$ of the DOX-loaded NP solution was injected subcutaneously into the abdomen of an anaesthetized Kunming mouse. The subcutaneous SERS spectrum detected using a $785 \mathrm{~nm}$ NIR laser excitation was due to the satisfactory biological tissue penetration ability. As indicated in Fig. 5e, the spectral signatures of DTDC could be clearly distinguished at the injection location, through the mouse skin, despite the intensity decreased to about half of that of the pure NP solution. These results proved the high sensitivity of the SERS labeling holding great potential to be used for nanomedicine tracking studies in animal models.

\subsection{Therapeutic property studies}

Next, we investigated the therapeutic properties of NPs and DOX-loaded NPs with and without laser irradiation via live/ dead cell staining. As shown in Fig. 6a, both the cells after
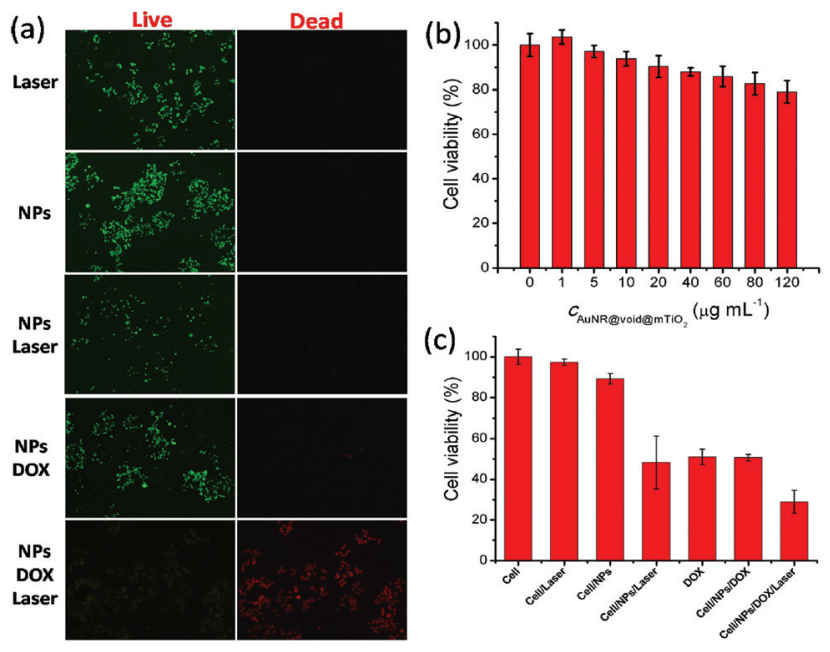

Fig. 6 (a) Live/dead cell monitoring of MCF-7 cells. All images collected after $60 \mathrm{~min}$ of incubation with 5-carboxyfluorescein diacetate/propidium iodide live/dead staining reagent. Cells showed a bright green fluorescence is an indication that the cells are alive. Cells exhibited neither red nor green fluorescence was an indication of an unhealthy state of cells. (b) Cell viability of AuNR@voidamTiO ${ }_{2} \mathrm{NPs}$ against MCF-7 cells at different concentrations for $24 \mathrm{~h}$. (c) Cell viabilities of MCF-7 cells in the presence of laser, blank NPs $\left(10 \mu \mathrm{g} \mathrm{mL}^{-1}\right)$, NPs upon laser irradiation and those incubated with $1 \mu \mathrm{M}$ DOX with and without irradiation. The power density of the $785 \mathrm{~nm}$ laser was $6.25 \mathrm{~W} \mathrm{~cm}^{-2}$ and the exposure time was $5 \mathrm{~min}$.

laser exposure $\left(6.25 \mathrm{~W} \mathrm{~cm}^{-2}\right)$ and incubation with blank NP $\left(10 \mu \mathrm{g} \mathrm{mL}^{-1}\right)$ showed bright green fluorescence and no red fluorescence, indicating that the employed laser power density and blank NP did not cause any cell death. In contrast, laser irradiation of cells incubated with blank NPs or uptake of DOX-loaded NPs resulted in decreased green fluorescence and sporadically distributed weak red fluorescence, indicating an unhealthy state of the cells. Laser irradiation of the cells with internalized DOX-loaded NPs resulted in severe cell death, as indicated by pale green and strong red fluorescence. These results preliminarily proved that cell death was more effective when cells were incubated with drug-loaded NPs and irradiated with laser compared to all other cases.

To further evaluate the effectiveness of therapy using this multifunctional platform, MTT assay was employed to quantify the viability of MCF-7 cells treated under different conditions. It was found that blank NPs exhibited little toxicity to cells even under high concentrations of $120 \mu \mathrm{g} \mathrm{mL}{ }^{-1}$ after 24 hours of incubation (Fig. 6b). Considering the NP concentration dependent cytotoxicity and DOX loading content, a NP concentration of $10 \mu \mathrm{g} \mathrm{mL}^{-1}$ was fixed for the following experiment. It could be seen that blank NPs incubation almost did not affect the cell viability (Fig. 6c). A suitable laser power density was also important, which should efficiently trigger the drug release and cell ablation functions at the tumor site without damaging normal cells. We applied a power density of $6.25 \mathrm{~W}$ $\mathrm{cm}^{-2}$ to treat the blank MCF-7 cells (without NP) for $5 \mathrm{~min}$ and no obvious cytotoxicity was observed after $24 \mathrm{~h}$ of incubation. Whereas the viability dramatically decreased to nearly $40.9 \%$ 
for NP filled cells upon NIR light irradiation, indicating the as expected strong photothermal killing ability of NP. Furthermore, we compared the cell killing efficacy free of DOX and DOX-loaded NPs with and without NIR light irradiation. At the DOX concentration of $1 \mu \mathrm{M}$, free of DOX and DOX-loaded NPs showed similar potency and about $50 \%$ of the cells were dead without light exposure. In comparison, DOX-loaded NPs under 5 min NIR irradiation killed $71.1 \%$ of the cells, illustrating an apparent chemo-photothermal synergistic effect.

\section{Conclusions}

We have successfully prepared novel yolk-shell AuNR@void@m $\mathrm{miO}_{2}$ NPs as theranostic tools for simultaneous SERS imaging and chemo-photothermal therapy. The interesting nanostructure, multifunction and terrific structural stability achieved taking advantage of multiple special features of AuNR, $\mathrm{mSiO}_{2}$ and $\mathrm{mTiO}_{2}$. Taking advantage of the novel and versatile "up to down" SERS labeling strategy, a bright SERS signal was obtained from NPs, which was successfully used for intracellular imaging and in vivo monitoring. Compared with chemotherapy or photothermal treatment, the combined treatment showed a synergistic effect, resulting in higher therapeutic efficacy. This proof-of-concept work aimed to provide a versatile Raman reporter and a drug co-loading strategy for developing SERS guided nanomedicine, and the NPs were directly used without surface functionalization before the cell culture and in vivo administration, namely, the interaction with a biological system was non-specific. We believe that if the NPs were PEGylated and targeting moieties labeled, the long circulation in the body and tumor targeting properties would be achieved, making the nanoplatform applicable for in vivo tumor SERS imaging and cancer treatment.

\section{Acknowledgements}

Financial support from the National Natural Science Foundation of China (81102415, 21305157 and 21275158), the Natural Science Foundation of Shandong Province of China (ZR2010BQ012), the Science and Technology Development Plan of Yantai (2011071), and the Scientific Research Foundation for the Returned Overseas Chinese Scholars State Education Ministry is gratefully acknowledged.

\section{Notes and references}

1 D. E. Lee, H. Koo, I. C. Sun, J. H. Ryu, K. Kim and I. C. Kwon, Chem. Soc. Rev., 2012, 41, 2656-2672.

2 Y. Q. Wang and L. X. Chen, Nanomed.: Nanotechnol. Biol. Med., 2011, 7, 385-402.

3 X. Yang, X. Liu, Z. Liu, F. Pu, J. Ren and X. Qu, Adv. Mater., 2012, 24, 2890-2895.

4 D. Lin, T. Qin, Y. Wang, X. Sun and L. Chen, ACS Appl. Mater. Interfaces, 2014, 6, 1320-1329.
5 M. P. Melancon, M. Zhou and C. Li, Acc. Chem. Res., 2011, 44, 947-956.

6 W. Fang, Z. Wang, S. Zong, H. Chen, D. Zhu, Y. Zhong and Y. Cui, Biosens. Bioelectron., 2014, 57, 10-15.

7 A. M. Fales, H. Yuan and T. Vo-Dinh, Langmuir, 2011, 27, 12186-12190.

8 K. V. Kong, Z. Lam, W. D. Goh, W. K. Leong and M. Olivo, Angew. Chem., Int. Ed., 2012, 51, 9796-9799.

9 Y. Zhang, J. Qian, D. Wang, Y. Wang and S. He, Angew. Chem., Int. Ed., 2013, 52, 1148-1151.

10 A. M. Fales, H. Yuan and T. Vo-Dinh, Mol. Pharm., 2013, 10, 2291-2298.

11 Y. Wang, B. Yan and L. Chen, Chem. Rev., 2013, 113, 13911428.

12 X. Niu, H. Chen, Y. Wang, W. Wang, X. Sun and L. Chen, ACS Appl. Mater. Interfaces, 2014, 6, 5152-5160.

13 H. Abramczyk and B. Brozek-Pluska, Chem. Rev., 2013, 113, 5766-5781.

14 S. Schlucker, Angew. Chem., Int. Ed., 2014, 53, 47564795.

15 J. Song, L. Pu, J. Zhou, B. Duan and H. Duan, ACS Nano, 2013, 7, 9947-9960.

16 G. von Maltzahn, A. Centrone, J. H. Park, R. Ramanathan, M. J. Sailor, T. A. Hatton and S. N. Bhatia, Adv. Mater., 2009, 21, 3175-3180.

17 L. Tian, N. Gandra and S. Singamaneni, ACS Nano, 2013, 7, 4252-4260.

18 W. T. Lu, A. K. Singh, S. A. Khan, D. Senapati, H. T. Yu and P. C. Ray, J. Am. Chem. Soc., 2010, 132, 18103-18114.

19 Z. Zhang, L. Wang, J. Wang, X. Jiang, X. Li, Z. Hu, Y. Ji, X. Wu and C. Chen, Adv. Mater., 2012, 24, 1418-1423.

20 S. Shen, H. Tang, X. Zhang, J. Ren, Z. Pang, D. Wang, H. Gao, Y. Qian, X. Jiang and W. Yang, Biomaterials, 2013, 34, 3150-3158.

21 J. Liu, S. Z. Qiao, J. S. Chen, X. W. Lou, X. R. Xing and G. Q. Lu, Chem. Commun., 2011, 47, 12578-12591.

22 K. C. Wu, Y. Yamauchi, C. Y. Hong, Y. H. Yang, Y. H. Liang, T. Funatsu and M. Tsunoda, Chem. Commun., 2011, 47, 5232-5234.

23 B. Nikoobakht and M. A. El-Sayed, Chem. Mater., 2003, 15, 1957-1962.

24 I. Gorelikov and N. Matsuura, Nano Lett., 2008, 8, 369-373.

25 A. F. Demirors, A. van Blaaderen and A. Imhof, Langmuir, 2010, 26, 9297-9303.

26 Y. Wang, L. Chen and P. Liu, Chem. - Eur. J., 2012, 18, 5935-5943.

27 A. F. Demirors, A. van Blaaderen and A. Imhof, Chem. Mater., 2009, 21, 979-984.

28 I. Lee, J. B. Joo, Y. Yin and F. Zaera, Angew. Chem., Int. Ed., 2011, 50, 10208-10211.

29 S. Kim, Y. Jang, W. K. Oh, C. Kim and J. Jang, Adv. Healthcare Mater., 2014, 3, 1097-1106.

30 L. Zhang, T. Wang, L. Yang, C. Liu, C. Wang, H. Liu, Y. A. Wang and Z. Su, Chem. - Eur. J., 2012, 18, 1251212521. 
31 L. M. Liz-Marzan, M. Giersig and P. Mulvaney, Langmuir, 1996, 12, 4329-4335.

32 G. B. Braun, S. J. Lee, T. Laurence, N. Fera, L. Fabris, G. C. Bazan, M. Moskovits and N. O. Reich, J. Phys. Chem. C, 2009, 113, 13622-13629.

33 M. Roca and A. J. Haes, J. Am. Chem. Soc., 2008, 130, 14273-14279.

34 J. Yang, D. Shen, L. Zhou, W. Li, J. Fan, A. M. El-Toni, W. X. Zhang, F. Zhang and D. Zhao, Adv. Healthcare Mater., 2014, 3, 1620-1628.
35 L.-L. Tay, P.-J. Huang, J. Tanha, S. Ryan, X. Wu, J. Hulse and L.-K. Chau, Chem. Commun., 2012, 48, 1024-1026.

36 C. Kim, S. Kim, W. K. Oh, M. Choi and J. Jang, Chem. - Eur. J., 2012, 18, 4902-4908.

37 L. B. Chen, F. Zhang and C. C. Wang, Small, 2009, 5, 621628.

38 K. Chen, J. Zhang and H. Gu, J. Mater. Chem., 2012, 22, 22005-22012.

39 S. Lee, M. S. Kim, D. Lee, T. K. Kwon, D. Khang, H. S. Yun and S. H. Kim, Int. J. Nanomedicine, 2013, 8, 147-158. 